Bangladesh J. Plant Taxon. 23(2): 143-160, 2016 (December)

(C) 2016 Bangladesh Association of Plant Taxonomists

\title{
UPDATED NOMENCLATURE AND TAXONOMIC STATUS OF THE PLANTS OF BANGLADESH INCLUDED IN HOOK. f. THE FLORA OF BRITISH INDIA: VOLUME III
}

\author{
M. EnAmur RASHid AND M. ATiQuR RAHMAN \\ Department of Botany, University of Chittagong, Chittagong-4331, Bangladesh
}

Keywords: J.D. Hooker; Flora of British India; Bangladesh; Nomenclature; Taxonomic Status.

\begin{abstract}
One hundred seventy seven species belonging to 88 genera under 14 natural orders are determined to have been recorded in the third volume of J.D. Hooker's, the Flora of British India from the area now fall in Bangladesh. These taxa are enumerated with updated nomenclature and current taxonomic status following ICN and Cronquist's system of plant classification respectively resulting in 169 species under 93 genera and 14 families. Collection locality with collector's name of each species wherever available, as cited in protologue, is also included.
\end{abstract}

\section{Introduction}

The plants from the area of Bangladesh included in volumes I and II of J.D. Hooker's, the Flora of British India have already been determined and reported with their updated nomenclature and taxonomic status following ICN (Rashid and Rahman, 2011, 2012). The present report deals with the treatment of the taxa of the volume III of the Flora of British India (1880-1882). This volume includes three parts (VII-IX) published in 3 different dates consisting of a total of 22 natural orders, 354 genera and 2174 species. In this volume, J.D. Hooker was assisted by an eminent botanist, C.B. Clarke. Hooker alone described 4 natural orders while Clarke alone described 18 natural orders.

Among these taxa, described in this volume, 177 species belonging to 88 genera and 14 natural orders are determined to be recorded from the area now in Bangladesh. The objective of the study was to update the nomenclature and taxonomic status of the plants of Bangladesh which have been included in the Flora of British India.

\section{Materials and Methods}

In this study, volume III of the Flora of British India (Hook.f., 1880-1882) has been surveyed for determining the taxa recorded from the area now fall in Bangladesh following Rashid and Rahman (2011, 2012). Bangladesh Gazetteers (Ishaq, 1979) has been consulted to ascertain the collection localities fall within the area of Bangladesh. In case of Bengal and Jainta hills, mentioned as collection localities, relevant literature, such as, Roxburgh $(1814,1820,1824,1832)$, Wallich (1828-1849), Kurz (1877), Prain (1903), Brandis (1906), Heinig (1925), Cowan (1926), Kanjilal et al. (1939), Rhaizada (1941) and Sinclair (1956) have been consulted to confirm whether the taxon belongs to the area now fall in Bangladesh.

The current nomenclature of each species was determined by consulting ICN (Voss, 1983; McNeill et al., 2012), Internet sourches (I-III), Brummitt and Powell (1992). Taxonomic status of the taxa were determined by following Cronquist (1981) and to determine the synonyms of respective species relevant literature, viz., Ali (1971), Hara and Williams (1979), Hara et al. (1982), Rahman and Wilcock (1991), Brummitt (1992), Wu and Raven (1994), Rahman and 
Wilcock (1995), Mabberley (1997), Press et al. (2000), Wu et al. (2005), Ahmed et al. (2008a, b; 2009a, b, c) and updated Kew Plant list from internet sources (I-III) have been consulted.

\section{Results and Discussion}

The search on the third volume of the Flora of the British India revealed a total of 177 species in 88 genera under 14 natural orders from the area now in Bangladesh (Table 1).

Table 1, Natural Orders with contributors and distribution of taxa in the Volume III

\begin{tabular}{lccc}
\hline $\begin{array}{l}\text { Natural order as in Hook.f. } \\
\text { (1880-1882) }\end{array}$ & Contributor & $\begin{array}{c}\text { Total No. of } \\
\text { genera/ species } \\
\text { described }\end{array}$ & $\begin{array}{c}\text { No. of genera/ species } \\
\text { from the area of } \\
\text { Bangladesh }\end{array}$ \\
\hline 1. Caprifoliaceae & C.B. Clarke & $8 / 49$ & $1 / 1$ \\
2. Rubiaceae & J.D. Hooker & $91 / 640$ & $31 / 81$ \\
3. Valerianeae & C.B. Clarke & $4 / 17$ & $0 / 0$ \\
4. Dipsaceae & C.B. Clarke & $4 / 17$ & $0 / 0$ \\
5. Compositae & J.D. Hooker & $123 / 634$ & $20 / 37$ \\
6. Stylidieae & C.B. Clarke & $1 / 3$ & $1 / 2$ \\
7. Goodenovieae & C.B. Clarke & $1 / 2$ & $0 / 0$ \\
8. Campanulaceae & C.B. Clarke & $13 / 65$ & $2 / 5$ \\
9. Vacciniaceae & C.B. Clarke & $4 / 50$ & $1 / 2$ \\
10. Ericaceae & C.B. Clarke & $9 / 65$ & $0 / 0$ \\
11. Monotropeae & C.B. Clarke & $3 / 3$ & $0 / 0$ \\
12.Epacrideae & C.B. Clarke & $1 / 1$ & $0 / 0$ \\
13. Diapensiaceae & C.B. Clarke & $1 / 1$ & $0 / 0$ \\
14. Plumbagineae & C.B. Clarke & $6 / 9$ & $1 / 1$ \\
15. Primulaceae & J.D. Hooker & $9 / 80$ & $2 / 2$ \\
16. Myrsineae & C.B. Clarke & $11 / 93$ & $4 / 6$ \\
17. Sapotaceae & C.B. Clarke & $8 / 55$ & $3 / 3$ \\
18. Ebenaceae & C.B. Clarke & $2 / 75$ & $1 / 7$ \\
19. Styraceae & C.B. Clarke & $2 / 70$ & $1 / 1$ \\
20. Oleaceae & C.B. Clarke & $10 / 91$ & $5 / 11$ \\
21. Salvadoraceae & C.B. Clarke & $3 / 5$ & $0 / 0$ \\
22. Apocynaceae & J.D. Hooker & $40 / 149$ & Genera/species $88 / 177$ \\
\hline Total: NO 22 & Contributors 02 & Genera/species & \\
\hline & 354/2174 & \\
\hline
\end{tabular}

After current nomenclatural treatment, the number of species reduced to 169, while the genera splited to 93. The species included in families Vaccinaceae and Styraceae have been transfered to Ericaceae and Symplocaceae respectively. It is determined, so far, that 29 generic names have been changed and 59 remain unchanged. On the other hand, 79 names of species have also been changed and 98 remain unchanged. Hence after updated nomenclatural treatment, 169 species and 93 genera under 14 families are recognized from the area of Bangladesh so far and presented in Table 2. 
Table 2. List of taxa as in Hook.f, the Flora of British India Volume III from the area of Bangladesh with their current nomenclature and taxonomic status

Species, Natural Order, recorded area - collector's name/ Wall. Cat. no. as in Hook.f. (1880-1882)

1. Sambucus javanica Blume

Natural Order: Caprifoliaceae

East Bengal - Not mentioned

2. Cephalanthus naucleoides DC.

Natural Order: Rubiaceae

Silhet - Not mentioned

3. Adina sessilifolia Hook.f.

Natural Order: Rubiaceae

Chittagong - Roxburgh \& c.

4. A. polycephala Benth.

Natural Order: Rubiaceae

Silhet - De Silva, Griffith

and Chittagong - J.D.H. \& T.T.

5. A. polycephala Benth. var. microphylla Hook.f.

Natural Order: Rubiaceae

Silhet -Wallich

6. Stephegyne diversifolia Hook.f.

Natural Order: Rubiaceae

Chittagong - Roxburgh \& c.

7. Nauclea ovalifolia Roxb.

Natural Order: Rubiaceae

Silhet - Not mentioned

8. Uncaria ovata $\mathrm{Br}$.

Natural Order: Rubiaceae

Silhet - Wall. Cat. 6112

9. U. sessilifructus Roxb.

Natural Order: Rubiaceae

Chittagong - Not mention

10. U. homomalla Miq.

Natural Order: Rubiaceae

Eastern Bengal; Jyntea hills - Wall. Cat. 6108

11. U. pilosa Roxb.

Natural Order: Rubiaceae

Chittagong - Roxburgh, J.D.H. \& T.T.

12. Hymenodictyon excelsum Wall.

Natural Order: Rubiaceae

Chittagong - Not mentioned
Current nomenclature with loc. cit. and family as of Cronquist (1981)

1. Sambucus javanica Reinw. ex Blume in Bijdr. Fl. Ned. Ind. 13: 657 (1825).

Family: Caprifoliaceae

2. Cephalanthus tetrandra (Roxb.) Ridsdale $\&$ Bakh.f. in Blumea 23: 182 (1976).

Family: Rubiaceae

3. Neonauclea sessilifolia (Roxb.) Merr. in J. Wash. Acad. Sci. 5: 542 (1915).

Family: Rubiaceae

4. Metadina trichotoma (Zoll. \& Mor.) Bakh.f., Taxon 19: 472 (1970).

Family: Rubiaceae

4. Metadina trichotoma (Zoll. \& Mor.) Bakh.f., Taxon 19: 472 (1970).

Family: Rubiaceae

5. Mitragyna diversifolia (Wall. ex G. Don) Havil. in J. Linn. Soc., Bot. 33: 71 (1897). Family:

Rubiaceae

3. Neonauclea sessilifolia (Roxb.) Merr. in

J. Wash. Acad. Sci. 5: 542 (1915).

Family: Rubiaceae

6. Uncaria canescens Korth., Verh. Nat. Gesch. Ned. Bot.: 172 (1842).

Family: Rubiaceae

7. Uncaria sessilifructus Roxb., Fl. Ind. 2: 130 (1824). Family: Rubiaceae

8. Uncaria homomalla Miq. in Fl. Ned. Ind. 2: 343 (1857). Family: Rubiaceae

9. Uncaria scandens (Smith) Hutch., Sarg. Pl. Wilson. 3: 406 (1916). Family: Rubiaceae

10. Hymenodictyon orixense (Roxb.) Mabb., Taxon 31: 66 (1982). Family: Rubiaceae 


\begin{tabular}{|c|c|}
\hline $\begin{array}{l}\text { Species, Natural Order, recorded area - collector’s } \\
\text { name/ Wall. Cat. no. as in Hook.f. (1880-1882) }\end{array}$ & $\begin{array}{l}\text { Current nomenclature with loc. cit. and family as of } \\
\text { Cronquist (1981) }\end{array}$ \\
\hline $\begin{array}{l}\text { 13. Wendlandia tinctoria DC. } \\
\text { Natural Order: Rubiaceae } \\
\text { Chittagong - Not mentioned }\end{array}$ & $\begin{array}{l}\text { 11. Wendlandia tinctoria (Roxb.) DC., Prodr. 4: } \\
411 \text { (1830). Family: Rubiaceae }\end{array}$ \\
\hline $\begin{array}{l}\text { 14. W. paniculata DC. } \\
\text { Natural Order: Rubiaceae } \\
\text { Silhet - Not mentioned }\end{array}$ & $\begin{array}{l}\text { 12. Wendlandia paniculata (Roxb.) DC., Prodr. } 4 \text { : } \\
411 \text { (1830). Family: Rubiaceae }\end{array}$ \\
\hline $\begin{array}{l}\text { 15. Dentella repens Forst. } \\
\text { Natural Order: Rubiaceae } \\
\text { Throughout Bengal - Not mentioned }\end{array}$ & $\begin{array}{l}\text { 13. Dentella repens J. R. Forst. \& G. Forst., Char. } \\
\text { Gen. Pl. Ins. Mar. Austr.: 26, t. } 13 \text { (1776). Family: } \\
\text { Rubiaceae }\end{array}$ \\
\hline $\begin{array}{l}\text { 16. Hedyotis scandens Roxb. } \\
\text { Natural Order: Rubiaceae } \\
\text { Silhet and Chittagong- Not mentioned }\end{array}$ & $\begin{array}{l}\text { 14. Hedyotis scandens Roxb., Fl. Ind. 1: } 369 . \\
\text { (1820). Family: Rubiaceae }\end{array}$ \\
\hline $\begin{array}{l}\text { 17. H. uncinella Hook. \& Arn. } \\
\text { Natural Order: Rubiaceae } \\
\text { Jyntea hills. - Wall. Cat. } 842\end{array}$ & $\begin{array}{l}\text { 15. Hedyotis uncinella Hook. \& Arn., Bot. } \\
\text { Beechey Voy.: } 192 \text { (1833). } \\
\text { Family: Rubiaceae }\end{array}$ \\
\hline $\begin{array}{l}\text { 18. H. auricularia Linn. } \\
\text { Natural Order: Rubiaceae } \\
\text { Chittagong - Not mentioned }\end{array}$ & $\begin{array}{l}\text { 16. Hedyotis auricularia L., Sp. Pl.: } 101 \text { (1753). } \\
\text { Family: Rubiaceae }\end{array}$ \\
\hline $\begin{array}{l}\text { 19. H. lineata Roxb. } \\
\text { Natural Order: Rubiaceae } \\
\text { Silhet and Chittagong - Not mentioned }\end{array}$ & $\begin{array}{l}\text { 17. Hedyotis lineata Roxb., Fl. Ind. 1: } 369 \text { (1820). } \\
\text { Family: Rubiaceae }\end{array}$ \\
\hline $\begin{array}{l}\text { 20. H. glabra Br. } \\
\text { Natural Order: Rubiaceae } \\
\text { Silhet - de Silva }\end{array}$ & $\begin{array}{l}\text { 18. Hedyotis insularis (Spreng.) Deb \& R.M. } \\
\text { Dutta in Taxon 32(2): } 285 \text { (1983). } \\
\text { Family: Rubiaceae }\end{array}$ \\
\hline $\begin{array}{l}\text { 21. H. hispida Retz } \\
\text { Natural Order: Rubiaceae } \\
\text { Chittagong - Not mentioned }\end{array}$ & $\begin{array}{l}\text { 19. Hedyotis verticillata (L.) Lam., Tabl. Encycl. } \\
\text { 1: } 271 \text { (1792). Family: Rubiaceae }\end{array}$ \\
\hline $\begin{array}{l}\text { 22. H. monocephala Br. } \\
\text { Natural Order: Rubiaceae } \\
\text { Silhet - Wall. Cat. } 846\end{array}$ & $\begin{array}{l}\text { 20. Hedyotis brunonis Merr. in Philipp. J. Sci. 60: } \\
35 \text { (1936). Family: Rubiaceae }\end{array}$ \\
\hline $\begin{array}{l}\text { 23. H. thomsoni Hook.f. } \\
\text { Natural Order: Rubiaceae } \\
\text { East Bengal - J.D.H. \& T.T. }\end{array}$ & $\begin{array}{l}\text { 21. Hedyotis thomsonii Hook.f., Fl. Brit. India 3: } \\
63 \text { (1880). Family: Rubiaceae }\end{array}$ \\
\hline $\begin{array}{l}\text { 24. Oldenlandia diffusa Roxb. var. extensa Hook.f. } \\
\text { Natural Order: Rubiaceae } \\
\text { Silhet - Wall. Cat. } 869 \text { \& Griffith }\end{array}$ & $\begin{array}{l}\text { 22. Hedyotis diffusa var. extensa (Hook.f.) Dutta } \\
\text { in Bot. Surv. India (2004). } \\
\text { Family: Rubiaceae }\end{array}$ \\
\hline $\begin{array}{l}\text { 25. O. crystallina Roxb. } \\
\text { Natural Order: Rubiaceae } \\
\text { East Bengal - Griffith, } \\
\text { Chittagong - C.B. Clarke }\end{array}$ & $\begin{array}{l}\text { 23. Hedyotis pumila L.f., Suppl. Pl.: } 119 \text { (1781). } \\
\text { Family: Rubiaceae }\end{array}$ \\
\hline
\end{tabular}




\begin{tabular}{|c|c|}
\hline $\begin{array}{c}\text { Species, Natural Order, recorded area - collector's } \\
\text { name/ Wall. Cat. no. as in Hook.f. (1880-1882) }\end{array}$ & $\begin{array}{l}\text { Current nomenclature with loc. cit. and family as of } \\
\text { Cronquist (1981) }\end{array}$ \\
\hline $\begin{array}{l}\text { 26. O. trinervia Retz. } \\
\text { Natural Order: Rubiaceae } \\
\text { Chittagong - J.D.H. \& T.T. }\end{array}$ & $\begin{array}{l}\text { 24. Hedyotis trinervia (Retz.) Roem \& Schult., } \\
\text { Syst. Veg. 3: } 197 \text { (1818). } \\
\text { Family: Rubiaceae }\end{array}$ \\
\hline $\begin{array}{l}\text { 27. O. paniculata Linn. } \\
\text { Natural Order: Rubiaceae } \\
\text { Silhet - Not mentioned }\end{array}$ & $\begin{array}{l}\text { 25. Hedyotis racemosa Lam., Encycl. 3: } 80 \\
\text { (1789). Family: Rubiaceae }\end{array}$ \\
\hline $\begin{array}{l}\text { 28. Anotis urophilla Wall. } \\
\text { Natural Order: Rubiaceae } \\
\text { Jyntea - Gomez, Griffith, \& c. }\end{array}$ & $\begin{array}{l}\text { 26. Neanotis urophylla (Wall. ex Wight \& Arn.) } \\
\text { W.H. Lewis, Ann. Missouri Bot. Gard. 53: } 40 \\
\text { (1966). Family: Rubiaceae }\end{array}$ \\
\hline $\begin{array}{l}\text { 29. Ophiorrhiza harrisiana Heyne } \\
\text { Natural Order: Rubiaceae } \\
\text { Silhet and Chittagong - Not mentioned }\end{array}$ & $\begin{array}{l}\text { 27. Ophiorrhiza rugosa Wall ex Roxb., Fl. Ind. 2: } \\
547 \text { (1824). Family: Rubiaceae }\end{array}$ \\
\hline $\begin{array}{l}\text { 30. O. harrisiana Heyne var. argentea Wall. } \\
\text { Natural Order: Rubiaceae } \\
\text { Silhet and Chittagong - Not mentioned }\end{array}$ & $\begin{array}{l}\text { 28. Ophiorrhiza rugosa var. argentea (Wall. ex } \\
\text { G. Don) Deb \& Mondal in Bull. Bot. Surv. India } 24 \\
\text { (1-4): } 228 \text { (1983). } \\
\text { Family: Rubiaceae }\end{array}$ \\
\hline $\begin{array}{l}\text { 31. O. trichocarpa Blume } \\
\text { Natural Order: Rubiaceae } \\
\text { Chittagong - Lister }\end{array}$ & $\begin{array}{l}\text { 29. Ophiorrhiza trichocarpos Blume, Bijdr.: } 977 \\
\text { (1826). Family: Rubiaceae }\end{array}$ \\
\hline $\begin{array}{l}\text { 32. O. Wallichii Hook.f. } \\
\text { Natural Order: Rubiaceae } \\
\text { Jyntea hills - Gomez }\end{array}$ & $\begin{array}{l}\text { 30. Ophiorrhiza wallichii Hook.f., Fl. Brit. India } \\
\text { 3: } 79 \text { (1880); Family: Rubiaceae }\end{array}$ \\
\hline $\begin{array}{l}\text { 33. O. villosa Roxb. } \\
\text { Natural Order: Rubiaceae } \\
\text { Chittagong hills - Roxburgh }\end{array}$ & $\begin{array}{l}\text { 31. Ophiorrhiza villosa Roxb., Fl. Ind. 2: } 546 \\
\text { (1824). Family: Rubiaceae }\end{array}$ \\
\hline $\begin{array}{l}\text { 34. Silvianthus bracteatus Hook.f. } \\
\text { Natural Order: Rubiaceae } \\
\text { Silhet - de Silva, Griffith \& c. }\end{array}$ & $\begin{array}{l}\text { 32. Silvianthus bracteatus Hook.f., Icon. Pl. t. } \\
\text { 1048: } 36 \text { (1868). } \\
\text { Family: Caprifoliaceae }\end{array}$ \\
\hline $\begin{array}{l}\text { 35. Mussaenda Roxburghii Hook.f. } \\
\text { Natural Order: Rubiaceae } \\
\text { Chittagong - J.D.H. \& T.T. }\end{array}$ & $\begin{array}{l}\text { 33. Mussaenda roxburghii Hook.f., Fl. Brit. India } \\
\text { 3: } 87 \text { (1880). Family: Rubiaceae }\end{array}$ \\
\hline $\begin{array}{l}\text { 36. M. glabra Vahl } \\
\text { Natural Order: Rubiaceae } \\
\text { Chittagong - Griffith \& Helfer }\end{array}$ & $\begin{array}{l}\text { 34. Mussaenda glabra Vahl, Symb. Bot. 3: } 38 \\
\text { (1794). Family: Rubiaceae }\end{array}$ \\
\hline $\begin{array}{l}\text { 37. Adenosacme longifolia Wall. } \\
\text { Natural Order: Rubiaceae } \\
\text { Chittagong - Not mentioned }\end{array}$ & $\begin{array}{l}\text { 35. Mycetia longifolia (Wall.) Kuntze, Revis. Gen. } \\
\text { Pl. 1: } 289 \text { (1891). Family: Rubiaceae }\end{array}$ \\
\hline $\begin{array}{l}\text { 38. Myrioneuron nutans Wall. } \\
\text { Natural Order: Rubiaceae } \\
\text { Chittagong hills - C.B. Clarke }\end{array}$ & $\begin{array}{l}\text { 36. Myrioneuron nutans Wall. ex Kurz, Fl. Brit. } \\
\text { Burma 2: } 55 \text { (1874). } \\
\text { Family: Rubiaceae }\end{array}$ \\
\hline
\end{tabular}




\begin{tabular}{ll}
\hline $\begin{array}{l}\text { Species, Natural Order, recorded area - collector’s } \\
\text { name/ Wall. Cat. no. as in Hook.f. (1880-1882) }\end{array}$ & $\begin{array}{c}\text { Current nomenclature with loc. cit. and family as of } \\
\text { Cronquist (1981) }\end{array}$ \\
\hline $\begin{array}{l}\text { 39. M.Clarkei Hook.f. } \\
\text { Natural Order: Rubiaceae }\end{array}$ & $\begin{array}{l}\text { 37. Myrioneuron clarkei Hook.f., Fl. Brit. India 3: } \\
\text { Chittagong - J.D.H. \& T.T. }\end{array}$ \\
$\begin{array}{l}\text { 40. Webera odorata Roxb. } \\
\text { Natural Order: Rubiaceae }\end{array}$ & $\begin{array}{l}\text { 38. Tarenna odorata (Roxb.) B. L. Rob., Proc. } \\
\text { Amer. Acad. Arts. 45: 405 (1910). Family: }\end{array}$ \\
Silhet - de Silva and Griffith & Rubiaceae \\
41. W. disperma Hook.f. & 39. Tarenna disperma (Hook.f.) Pitard in Fl. Gen. \\
Natural Order: Rubiaceae & Indo-China 3: 208 (1923). \\
Silhet - Griffith and J.D.H. \& T.T. & Family: Rubiaceae
\end{tabular}

42. W. Campaniflora Hook.f.

Natural Order: Rubiaceae

Chittagong-Bruce, Seetakoond - J.D.H. \& T.T. and

Burkul - C.B. Clarke

43. Randia tetrasperma Roxb.

Natural Order: Rubiaceae

Silhet - Not mentioned

44. R. fasciculata DC.

Natural Order: Rubiaceae

Silhet - Wallich \& c.

45. $R$. dumetorum Lamk.

Natural Order: Rubiaceae

Chittagong and Silhet - Not mentioned

40. Tarenna campaniflora (Hook.f.) Balak., Bull. Bot. Surv. India 22 (1-4): 175 (1982). Family:

Rubiaceae

41. Himalrandia tetrasperma (Roxb.) Yamazaki in Jap. J. Bot. 45: 340 (1970). Family: Rubiaceae

42. Benkara fasciculata (Roxb.) Ridsdale, Reinwardtia 12: 298 (2008).

Family: Rubiaceae

43. Catunaregam spinosa (Thunb.) Tirveng. in Bull. Mus. Natl. Hist. Nat., Ser. 3, Bot. 35: 13 (1978). Family: Rubiaceae

44. Oxyceros longiflorus (Lam.) T. Yamaz. in J. Jap. Bot. 45: 339 (1970).

Family: Rubiaceae

45. Tarennoidea wallichii (Hook.f.) Tirveng. \& Sastre in Mauritius Inst. Bull. 8(4): 90 (1979).

Family: Rubiaceae

46. Gardenia resinifera Roth, Nov. Pl. Sp.: 150 (1821). Family: Rubiaceae

Chittagong - Roxburgh \& c.

49. G. coronaria Ham.

Natural Order: Rubiaceae

Chittagong - Roxburgh \& c.

50. G. turgida Roxb.

Natural Order: Rubiaceae

Silhet - Not mentioned

51. G. campanulata Roxb.

Natural Order: Rubiaceae

Chittagong and Silhet - Roxburgh, Griffith \& c.

47. Gardenia coronaria Buch.-Ham. in Embassy Ava ed. 2, 3: 307 (1809).

Family: Rubiaceae

48. Ceriscoides turgida (Roxb.) Tirveng., Bull. Mus. Natl. Hist. Nat., Ser. 3, Bot. 35: 15 (1978).

Family: Rubiaceae

49. Ceriscoides campanulata (Roxb.) Tirveng, Bull. Mus. Natl. Hist. Nat., Ser. 3, Bot. 35: 16 (1978). Family: Rubiaceae 


\begin{tabular}{|c|c|}
\hline $\begin{array}{l}\text { Species, Natural Order, recorded area - collector’s } \\
\text { name/ Wall. Cat. no. as in Hook.f. (1880-1882) }\end{array}$ & $\begin{array}{l}\text { Current nomenclature with loc. cit. and family as of } \\
\text { Cronquist (1981) }\end{array}$ \\
\hline $\begin{array}{l}\text { 52. Petunga Roxburghii DC. } \\
\text { Natural Order: Rubiaceae } \\
\text { Chittagong and Silhet - Roxburgh \& Wallich }\end{array}$ & $\begin{array}{l}\text { 50. Hypobathrum racemosum (Roxb.) Kurz, } \\
\text { Prelim. Rep. Forest Pegu App. B: } 59 \text { (1875). } \\
\text { Family: Rubiaceae }\end{array}$ \\
\hline $\begin{array}{l}\text { 53. Hyptianthera stricta W. \& A. } \\
\text { Natural Order: Rubiaceae } \\
\text { E. Bengal - Not mentioned }\end{array}$ & $\begin{array}{l}\text { 51. Hyptianthera stricta (Roxb. ex Schult.) Wight } \\
\text { \& Arn., Prodr. Fl. Ind. Orient. } 399 \text { (1834). Family: } \\
\text { Rubiaceae }\end{array}$ \\
\hline $\begin{array}{l}\text { 54. Canthium didymum Roxb. } \\
\text { Natural Order: Rubiaceae } \\
\text { Jyntea - de Silva }\end{array}$ & $\begin{array}{l}\text { 52. Canthium dicoccum (Gaertn.) Teijsm. \& } \\
\text { Binn., Cat. Herb. Bogor: } 113 \text { (1866). } \\
\text { Family: Rubiaceae }\end{array}$ \\
\hline $\begin{array}{l}\text { 55. C. angustifolium Roxb. } \\
\text { Natural Order: Rubiaceae } \\
\text { Sunderbunds, Chittagong and Silhet - Roxburgh \& c. }\end{array}$ & $\begin{array}{l}\text { 53. Canthium angustifolium Roxb., Fl. Ind. 2: } \\
169 \text { (1824). } \\
\text { Family: Rubiaceae }\end{array}$ \\
\hline $\begin{array}{l}\text { 56. C. parvifolium Roxb. } \\
\text { Natural Order: Rubiaceae } \\
\text { Chittagong - Wall. Cat. } 8257 \text { and J.D.H. \& T.T. }\end{array}$ & $\begin{array}{l}\text { 54. Canthium parvifolium Roxb., Fl. Ind. 2: } 170 \\
\text { (1824). } \\
\text { Family: Rubiaceae }\end{array}$ \\
\hline $\begin{array}{l}\text { 57. Ixora acuminata Roxb. } \\
\text { Natural Order: Rubiaceae } \\
\text { Chittagong - C.B. Clarke }\end{array}$ & $\begin{array}{l}\text { 55. Ixora acuminata Roxb., Fl. Ind. 1: } 383 \text { (1820). } \\
\text { Family: Rubiaceae }\end{array}$ \\
\hline $\begin{array}{l}\text { 58. I. parviflora Vahl } \\
\text { Natural Order: Rubiaceae } \\
\text { Chittagong - C.B. Clarke }\end{array}$ & $\begin{array}{l}\text { 56. Ixora pavetta Andr., Bot. Repos. 2: t } 78 \\
\text { (1799). } \\
\text { Family: Rubiaceae }\end{array}$ \\
\hline $\begin{array}{l}\text { 59. I. villosa Roxb. } \\
\text { Natural Order: Rubiaceae } \\
\text { Silhet - Wall. Cat. } 6137\end{array}$ & $\begin{array}{l}\text { 57. Ixora balakrishnii Deb \& Rout in J. Bombay } \\
\text { Nat. Hist. Soc. 89: } 44 \text { (1992). } \\
\text { Family: Rubiaceae }\end{array}$ \\
\hline $\begin{array}{l}\text { 60. I. cuneifolia Roxb. } \\
\text { Natural Order: Rubiaceae } \\
\text { Silhet - De Silva }\end{array}$ & $\begin{array}{l}\text { 58. Ixora cuneifolia Roxb., Fl. Ind. 1: } 380 \text { (1820). } \\
\text { Family: Rubiaceae }\end{array}$ \\
\hline $\begin{array}{l}\text { 61. I. coccinea Linn. } \\
\text { Natural Order: Rubiaceae } \\
\text { Chittagong - J.D.H. \& T. T. }\end{array}$ & $\begin{array}{l}\text { 59. Ixora coccinea L., Sp. Pl.: } 110 \text { (1753). } \\
\text { Family: Rubiaceae }\end{array}$ \\
\hline $\begin{array}{l}\text { 62. Pavetta subcapita Hook.f. } \\
\text { Natural Order: Rubiaceae } \\
\text { Jyntea hills - Gomez }\end{array}$ & $\begin{array}{l}\text { 60. Pavetta subcapita Wall. ex Hook.f., Fl. Brit. } \\
\text { India 3: } 150 \text { (1880). } \\
\text { Family: Rubiaceae }\end{array}$ \\
\hline $\begin{array}{l}\text { 63. P. naucleiflora Wall. } \\
\text { Natural Order: Rubiaceae } \\
\text { Silhet - Wall. Cat. } 6171\end{array}$ & $\begin{array}{l}\text { 61. Pavetta naucleiflora R. Br. ex G. Don, Gen. } \\
\text { Hist. 3: } 575 \text { (1834). } \\
\text { Family: Rubiaceae }\end{array}$ \\
\hline $\begin{array}{l}\text { 64. Coffea bengalensis Roxb. } \\
\text { Natural Order: Rubiaceae } \\
\text { Silhet and Chittagong - Not mentioned }\end{array}$ & $\begin{array}{l}\text { 62. Psilanthus bengalensis (Roxb. ex Schult.) } \\
\text { Leroy, Bull. Mus. Natl. Hist. Nat., B, Adansonia 3: } \\
252 \text { (1982). } \\
\text { Family: Rubiaceae }\end{array}$ \\
\hline
\end{tabular}




\begin{tabular}{|c|c|}
\hline $\begin{array}{l}\text { Species, Natural Order, recorded area - collector’s } \\
\text { name/ Wall. Cat. no. as in Hook.f. (1880-1882) }\end{array}$ & $\begin{array}{l}\text { Current nomenclature with loc. cit. and family as of } \\
\text { Cronquist (1981) }\end{array}$ \\
\hline $\begin{array}{l}\text { 65. C. fragrans Wall. } \\
\text { Natural Order: Rubiaceae } \\
\text { Silhet - Gomez }\end{array}$ & $\begin{array}{l}\text { 63. Psilanthus fragrans (Wall. ex Hook.f.) Leroy, } \\
\text { Bull. Mus. Natl. Hist. Nat., B, Adansonia 3: } 256 \\
\text { (1982). } \\
\text { Family: Rubiaceae }\end{array}$ \\
\hline $\begin{array}{l}\text { 66. C. khasiana Hook.f. } \\
\text { Natural Order: Rubiaceae } \\
\text { Jyntea hill - J.D.H. \& T.T. \& C.B. Clarke }\end{array}$ & $\begin{array}{l}\text { 64. Nostolachma khasiana (Korth.) Deb \& Lahiri, } \\
\text { Bull. Bot. Surv. India 17: } 162 \text { (1978). } \\
\text { Family: Rubiaceae }\end{array}$ \\
\hline $\begin{array}{l}\text { 67. Morinda angustifolia Roxb. } \\
\text { Natural Order: Rubiaceae } \\
\text { Chittagong - Not mentioned }\end{array}$ & $\begin{array}{l}\text { 65. Morinda angustifolia Roxb., Pl. Coromandel } \\
\text { 3: } 32 \text { (1815). } \\
\text { Family: Rubiaceae }\end{array}$ \\
\hline $\begin{array}{l}\text { 68. M. persicaefolia Ham. } \\
\text { Natural Order: Rubiaceae } \\
\text { Chittagong - Hamilton }\end{array}$ & $\begin{array}{l}\text { 66. Morinda persicaefolia Buch.-Ham., Trans. } \\
\text { Linn. Soc. London 13: } 535 \text { (1822). } \\
\text { Family: Rubiaceae }\end{array}$ \\
\hline $\begin{array}{l}\text { 69. M. umbellata Linn. } \\
\text { Natural Order: Rubiaceae } \\
\text { East Bengal - Not mentioned }\end{array}$ & $\begin{array}{l}\text { 67. Morinda umbellata L., Sp. Pl.: } 176 \text { (1753). } \\
\text { Family: Rubiaceae }\end{array}$ \\
\hline $\begin{array}{l}\text { 70. Psychotria adenophylla Wall. } \\
\text { Natural Order: Rubiaceae } \\
\text { Chittagong - J.D.H. \& T.T. }\end{array}$ & $\begin{array}{l}\text { 68. Psychotria adenophylla Wall. in Roxb., Fl. } \\
\text { Ind. 2: } 166 \text { (1824). } \\
\text { Family: Rubiaceae }\end{array}$ \\
\hline $\begin{array}{l}\text { 71. P. calocarpa Kurz } \\
\text { Natural Order: Rubiaceae } \\
\text { Chittagong - Gomez, \& c. }\end{array}$ & $\begin{array}{l}\text { 69. Psychotria calocarpa Kurz in J. Asiat. Soc. } \\
\text { Bengal, Pt. 2, Nat. Hist. 41(2): } 315 \text { (1872). } \\
\text { Family: Rubiaceae }\end{array}$ \\
\hline $\begin{array}{l}\text { 72. P. silhetensis Hook.f. } \\
\text { Natural Order: Rubiaceae } \\
\text { Silhet - de Silva \& c. }\end{array}$ & $\begin{array}{l}\text { 70. Psychotria silhetensis Hook.f., Fl. Brit. India } \\
\text { 3: } 174 \text { (1880). Family: Rubiaceae }\end{array}$ \\
\hline $\begin{array}{l}\text { 73. P. Montana Blume } \\
\text { Natural Order: Rubiaceae } \\
\text { Silhet - De Silva }\end{array}$ & $\begin{array}{l}\text { 71. Psychotria montana Blume, Catalogus: } 54 \\
\text { (1823). Family: Rubiaceae }\end{array}$ \\
\hline $\begin{array}{l}\text { 74. P. sphaerocarpa Wall. } \\
\text { Natural Order: Rubiaceae } \\
\text { Silhet hills - Wallich }\end{array}$ & $\begin{array}{l}\text { 72. Psychotria sphaerocarpa Wall. in Roxb., Fl. } \\
\text { Ind. 2: } 161 \text { (1820). Family: Rubiaceae }\end{array}$ \\
\hline $\begin{array}{l}\text { 75. Chasalia curviflora Thw. var ellipsoidea } \\
\text { Hook.f. }\end{array}$ & $\begin{array}{l}\text { 73. Chasalia curviflora Thw. var. ellipsoidea } \\
\text { Hook.f., Fl. Brit. India 3: } 177 \text { (1880). }\end{array}$ \\
\hline $\begin{array}{l}\text { Natural Order: Rubiaceae } \\
\text { Jyntea - C.B. Clarke }\end{array}$ & Family: Rubiaceae \\
\hline $\begin{array}{l}\text { 76. Geophila reniformis Don } \\
\text { Natural Order: Rubiaceae } \\
\text { Silhet - Roxburgh and De Silva }\end{array}$ & $\begin{array}{l}\text { 74. Geophila repens (L.) Johnst., Sargentia 8: } 281 \\
\text { (1949). Family: Rubiaceae }\end{array}$ \\
\hline $\begin{array}{l}\text { 77. Lasianthus cyanocarpus Jack } \\
\text { Natural Order: Rubiaceae } \\
\text { Silhet and Chittagong - Wallich \& c. }\end{array}$ & $\begin{array}{l}\text { 75. Lasianthus cyanocarpus Jack in Trans. Linn. } \\
\text { Soc. London 14: } 125 \text { (1823). } \\
\text { Family: Rubiaceae }\end{array}$ \\
\hline
\end{tabular}




\begin{tabular}{|c|c|}
\hline $\begin{array}{l}\text { Species, Natural Order, recorded area - collector's } \\
\text { name/ Wall. Cat. no. as in Hook.f. (1880-1882) }\end{array}$ & $\begin{array}{l}\text { Current nomenclature with loc. cit. and family as of } \\
\text { Cronquist (1981) }\end{array}$ \\
\hline $\begin{array}{l}\text { 78. L. wallichii Wight } \\
\text { Natural Order: Rubiaceae } \\
\text { Silhet - De Silva \& c. }\end{array}$ & $\begin{array}{l}\text { 76. Lasianthus attenuatus Jack in Trans. Linn. } \\
\text { Soc. London 14: } 126 \text { (1823). } \\
\text { Family: Rubiaceae }\end{array}$ \\
\hline $\begin{array}{l}\text { 79. L. tentaculus Hook.f. } \\
\text { Natural Order: Rubiaceae } \\
\text { Silhet -Wall. Cat. } 8306\end{array}$ & $\begin{array}{l}\text { 77. Lasianthus rigidus Miq., Fl. Ned. Ind. 2: } 321 \\
\text { (1857). Family: Rubiaceae }\end{array}$ \\
\hline $\begin{array}{l}\text { 80. L. attenuates Jack } \\
\text { Natural Order: Rubiaceae } \\
\text { Silhet - De Silva }\end{array}$ & $\begin{array}{l}\text { 76. Lasianthus attenuatus Jack in Trans. Linn. } \\
\text { Soc. London 14: } 126 \text { (1823). } \\
\text { Family: Rubiaceae }\end{array}$ \\
\hline $\begin{array}{l}\text { 81. L. tubiferus Hook.f. } \\
\text { Natural Order: Rubiaceae } \\
\text { Jyntea hills - Griffith, \& c. }\end{array}$ & $\begin{array}{l}\text { 78. Lasianthus inodorus Blume, Bijdr.: } 998 \\
\text { (1826). } \\
\text { Family: Rubiaceae }\end{array}$ \\
\hline $\begin{array}{l}\text { 82. L. inconspicuus Hook.f. } \\
\text { Natural Order: Rubiaceae } \\
\text { Silhet - Wallich; Wall. Cat. 8313L }\end{array}$ & $\begin{array}{l}\text { 79. Lasianthus lucidus var. inconspicuus (Hook.f.) } \\
\text { H. Zhu, Acta Bot. Yunnan. 20: } 154 \text { (1998). } \\
\text { Family: Rubiaceae }\end{array}$ \\
\hline $\begin{array}{l}\text { 83. Ethulia conyzoides Linn. } \\
\text { Natural Order: Compositae } \\
\text { Silhet- Not mentioned }\end{array}$ & $\begin{array}{l}\text { 80. Ethulia conyzoides L., Sp. Pl. ed. 2: } 1171 \\
\text { (1762). } \\
\text { Family: Asteraceae }\end{array}$ \\
\hline $\begin{array}{l}\text { 84. Vernonia Thomsoni Hook.f. } \\
\text { Natural Order: Compositae } \\
\text { Chittagong, Seetakoond - J.D.H. \& T. T. }\end{array}$ & $\begin{array}{l}\text { 81. Vernonia thomsonii Hook.f. Fl. Brit. India 3: } \\
232 \text { (1881). } \\
\text { Family: Asteraceae }\end{array}$ \\
\hline $\begin{array}{l}\text { 85. V. saligna DC. } \\
\text { Natural Order: Compositae } \\
\text { Chittagong - Not mentioned }\end{array}$ & $\begin{array}{l}\text { 82. Vernonia saligna DC., Prodr. 5: } 33 \text { (1836). } \\
\text { Family: Asteraceae }\end{array}$ \\
\hline $\begin{array}{l}\text { 86. V. arborea Ham. } \\
\text { Natural Order: Compositae } \\
\text { Silhet - Not mentioned }\end{array}$ & $\begin{array}{l}\text { 83. Vernonia arborea Buch.-Ham. ex Buch.- } \\
\text { Ham., Trans. Linn. Soc. London 14: } 218 \text { (1825). } \\
\text { Family: Asteraceae }\end{array}$ \\
\hline $\begin{array}{l}\text { 87. V. volkameriaefolia DC. } \\
\text { Natural Order: Compositae } \\
\text { Jaintea hills - Griffith }\end{array}$ & $\begin{array}{l}\text { 84. Vernonia volkameriaefolia DC., Prodr. 5: } 32 \\
\text { (1836). } \\
\text { Family: Asteraceae }\end{array}$ \\
\hline $\begin{array}{l}\text { 88. V. scandens DC. } \\
\text { Natural Order: Compositae } \\
\text { Silhet - Not mentioned }\end{array}$ & $\begin{array}{l}\text { 85. Vernonia vagans DC., Prodr. 5: } 32 \text { (1836). } \\
\text { Family: Asteraceae }\end{array}$ \\
\hline $\begin{array}{l}\text { 89. Cyathocline lyrata Cass. } \\
\text { Natural Order: Compositae } \\
\text { Chittagong - Not mentioned }\end{array}$ & $\begin{array}{l}\text { 86. Cyathocline purpurea (Buch.-Ham. ex D. } \\
\text { Don) Kuntze, Rev. Gen. Pl.: } 333 \text { (1891). } \\
\text { Family: Asteraceae }\end{array}$ \\
\hline $\begin{array}{l}\text { 90. Erigeron asteroides Roxb. } \\
\text { Natural Order: Compositae } \\
\text { Bengal - Not mentioned }\end{array}$ & $\begin{array}{l}\text { 87. Erigeron sublyratus Roxb. ex DC. in Wight, } \\
\text { Contr. Bot. Ind.: } 9 \text { (1834). } \\
\text { Family: Asteraceae }\end{array}$ \\
\hline
\end{tabular}




\begin{tabular}{|c|c|}
\hline $\begin{array}{l}\text { Species, Natural Order, recorded area - collector’s } \\
\text { name/ Wall. Cat. no. as in Hook.f. (1880-1882) }\end{array}$ & $\begin{array}{l}\text { Current nomenclature with loc. cit. and family as of } \\
\text { Cronquist (1981) }\end{array}$ \\
\hline 91. Conyza semipinnatifida Wall. & 88. Conyza semipinnatifida Wall. ex DC., Prodr. \\
\hline Natural Order: Compositae & 5: 382 (1836) \\
\hline Soonderbunds at Burisal - Clarke & Family: Asteraceae \\
\hline 92. Thespis divaricata DC. & 89. Thespis divaricata DC. in Guill. Arch. Bot. 2: \\
\hline Natural Order: Compositae & 517 (1833). \\
\hline Silhet - Not mentioned & Family: Asteraceae \\
\hline 93. Blumea amplectens DC. & 90. Blumea obliqua (L.) Druce, Rep. Bot. Excu. \\
\hline Natural Order: Compositae & Club Brit. Isles 4: 609 (1917). \\
\hline Bengal - Not mentioned & Family: Asteraceae \\
\hline 94. B. amplectens DC. var. maritima Hook.f. & 90. Blumea obliqua (L.) Druce, Rep. Bot. Excu. \\
\hline Natural Order: Compositae & Club Brit. Isles 4: 609 (1917). \\
\hline Soonderbunds - Not mentioned & Family: Asteraceae \\
\hline 95. Blumea bifoliata DC. & 91. Blumea bifoliata (L.) DC. in Wight, Contr. \\
\hline Natural Order: Compositae & Bot. Ind.: 14 (1834). \\
\hline Bengal - Not mentioned & Family: Asteraceae \\
\hline 96. B. sericans Hook.f. & 92. Blumea sericans (Kurz) Hook.f., Fl. Brit. India \\
\hline Natural Order: Compositae & 3: 262 (1881). \\
\hline Chittagong hills - Clarke & Family: Asteraceae \\
\hline 97. B. laciniata DC. & 93. Blumea laciniata (Roxb.) DC., Prodr. 5: 436 \\
\hline Natural Order: Compositae & (1836). \\
\hline Bengal - Not mentioned & Family: Asteraceae \\
\hline 98. B. oxyodonta DC. & 94. Blumea oxyodonta DC. in Wight, Contr. Bot. \\
\hline Natural Order: Compositae & Ind.: 15 (1834). \\
\hline Bengal - Not mentioned & Family: Asteraceae \\
\hline 99. B. myriocephala DC. & 95. Blumea lanceolaria (Roxb.) Druce, Bot. Soc. \\
\hline Natural Order: Compositae & Exch. Club Br. Isles 4: 609 (1917). \\
\hline Chittagong - Clarke & Family: Asteraceae \\
\hline 100. B. balsamifera DC. & 96. Blumea balsamifera (L.) DC., Prodr. 5: 447 \\
\hline Natural Order: Compositae & (1836). \\
\hline Chittagong - Not mentioned & Family: Asteraceae \\
\hline 101. Laggera flava Benth. & 97. Blumeopsis falcata (D. Don) Merr. in J. \\
\hline Natural Order: Compositae & Arnold Arbor. Cambridge (1938). \\
\hline Chittagong - Not mentioned & Family: Asteraceae \\
\hline 102. L. aurita Schultz-Bip. & 98. Blumea viscosa (Mill.) V. M. Badillo, Revista \\
\hline Natural Order: Compositae & Fac. Agron. (Maracay): 79 (1974). \\
\hline Chittagong - Not mentioned & Family: Asteraceae \\
\hline 103. Pluchea indica Less. & 99. Pluchea indica (L.) Less., Linnaea 6: 150 \\
\hline Natural Order: Compositae & (1831). \\
\hline Sunderbunds - Not mentioned & Family: Asteraceae \\
\hline
\end{tabular}




\begin{tabular}{|c|c|}
\hline $\begin{array}{l}\text { Species, Natural Order, recorded area - collector's } \\
\text { name/ Wall. Cat. no. as in Hook.f. (1880-1882) }\end{array}$ & $\begin{array}{c}\text { Current nomenclature with loc. cit. and family as of } \\
\text { Cronquist (1981) }\end{array}$ \\
\hline $\begin{array}{l}\text { 104. Sphaeranthus africanus Linn. } \\
\text { Natural Order: Compositae } \\
\text { Silhet - Not mentioned }\end{array}$ & $\begin{array}{l}\text { 100. Sphaeranthus africanus L., Sp. Pl. ed. 2: } \\
1314 \text { (1762). } \\
\text { Family: Asteraceae }\end{array}$ \\
\hline $\begin{array}{l}\text { 105. S. indicus Linn. } \\
\text { Natural Order: Compositae } \\
\text { Silhet - Not mentioned }\end{array}$ & $\begin{array}{l}\text { 101. Sphaeranthus indicus L., Sp. Pl.: } 927 \text { (1753). } \\
\text { Family: Asteraceae }\end{array}$ \\
\hline $\begin{array}{l}\text { 106. Caesulia axillaris Roxb. } \\
\text { Natural Order: Compositae } \\
\text { Chittagong - Not mentioned }\end{array}$ & $\begin{array}{l}\text { 102. Caesulia axillaris Roxb., Pl. Corom. 1: 64, t. } \\
93 \text { (1798). } \\
\text { Family: Asteraceae }\end{array}$ \\
\hline $\begin{array}{l}\text { 107. Enhydra fluctuans Lour. } \\
\text { Natural Order: Compositae } \\
\text { Silhet - Not mentioned }\end{array}$ & $\begin{array}{l}\text { 103. Enhydra fluctuans Lour., Fl. Cochinch. } 511 \\
\text { (1790). } \\
\text { Family: Asteraceae }\end{array}$ \\
\hline $\begin{array}{l}\text { 108. Wedelia calendulacea Less. } \\
\text { Natural Order: Compositae } \\
\text { Silhet - Not mentioned }\end{array}$ & $\begin{array}{l}\text { 104. Wedelia chinensis (Osbeck) Merr., Philipp. J. } \\
\text { Sci. 12: } 111 \text { (1917). } \\
\text { Family: Asteraceae }\end{array}$ \\
\hline $\begin{array}{l}\text { 109. W. biflora DC. } \\
\text { Natural Order: Compositae }\end{array}$ & $\begin{array}{l}\text { 105. Melanthera biflora (L.) Wild, Kirkia: } 54 \\
\text { (1965). } \\
\text { Family: Asteraceae }\end{array}$ \\
\hline $\begin{array}{l}\text { 110. Cotula hemisphaerica Wall. } \\
\text { Natural Order: Compositae } \\
\text { Dry rice field in Bengal - Not mentioned }\end{array}$ & $\begin{array}{l}\text { 106. Cotula hemisphaerica Wall. ex Benth. \& } \\
\text { Hook.f. Gen. Pl. 2: } 429 \text { (1873). } \\
\text { Family: Asteraceae }\end{array}$ \\
\hline $\begin{array}{l}\text { 111. Artemisia caruifolia Ham. } \\
\text { Natural Order: Compositae } \\
\text { Eastern Bengal - Not mentioned }\end{array}$ & $\begin{array}{l}\text { 107. Artemisia carvifolia Buch.-Ham. ex Roxb., } \\
\text { Fl. Ind. 2: } 422 \text { (1820). } \\
\text { Family: Asteraceae }\end{array}$ \\
\hline $\begin{array}{l}\text { 112. Senecio obtusatus Wall. } \\
\text { Natural Order: Compositae } \\
\text { Jyntea hills - De Silva }\end{array}$ & $\begin{array}{l}\text { 108. Senecio obtusatus Wall. ex DC., Prodr. 6: } \\
367 \text { (1838). } \\
\text { Family: Asteraceae }\end{array}$ \\
\hline $\begin{array}{l}\text { 113. S. ramosus Wall. } \\
\text { Natural Order: Compositae } \\
\text { Silhet - Roxburgh }\end{array}$ & $\begin{array}{l}\text { 109. Senecio ramosus Wall. ex Hook.f., Fl. Brit. } \\
\text { India 3: } 342 \text { (1881). } \\
\text { Family: Asteraceae }\end{array}$ \\
\hline $\begin{array}{l}\text { 114. Cnicus arvensis Hoffm. } \\
\text { Natural Order: Compositae } \\
\text { Soonderbunds - Not mentioned }\end{array}$ & $\begin{array}{l}\text { 110. Cirsium arvense (L.) Scop., Fl. Carn. ed. } 2 \\
\text { (2): } 126 \text { (1772). } \\
\text { Family: Asteraceae }\end{array}$ \\
\hline $\begin{array}{l}\text { 115. Saussurea affinis Spreng } \\
\text { Natural Order: Compositae } \\
\text { Silhet - Not mentioned }\end{array}$ & $\begin{array}{l}\text { 111. Hemistepta lyrata (Bunge) Bunge in Dorp. } \\
\text { Jahrb. Litt. 1: } 222 \text { (1833). } \\
\text { Family: Asteraceae }\end{array}$ \\
\hline $\begin{array}{l}\text { 116. Crepis acaulis Hook.f. } \\
\text { Natural Order: Compositae } \\
\text { Dinagepore - Not mentioned }\end{array}$ & $\begin{array}{l}\text { 112. Launaea acaulis (Roxb.) Kerr in Craib, Fl. } \\
\text { Siam. Enum. 2: } 299 \text { (1936). } \\
\text { Family: Asteraceae }\end{array}$ \\
\hline
\end{tabular}




\begin{tabular}{|c|c|}
\hline $\begin{array}{l}\text { Species, Natural Order, recorded area - collector's } \\
\text { name/ Wall. Cat. no. as in Hook.f. (1880-1882) }\end{array}$ & $\begin{array}{l}\text { Current nomenclature with loc. cit. and family as of } \\
\text { Cronquist (1981) }\end{array}$ \\
\hline $\begin{array}{l}\text { 117. Lectuca polycephala Benth. } \\
\text { Natural Order: Compositae } \\
\text { Bengal - Not mentioned }\end{array}$ & $\begin{array}{l}\text { 113. Ixeris polycephala Cass., Dict. Sci. Nat. 24: } \\
50 \text { (1822). } \\
\text { Family: Asteraceae }\end{array}$ \\
\hline $\begin{array}{l}\text { 118. Launaea aspleniifolia DC. } \\
\text { Natural Order: Compositae } \\
\text { Soonderbunds - Not mentioned }\end{array}$ & $\begin{array}{l}\text { 114. Launaea aspleniifolia (Willd.) DC., Prodr. } 7 \text { : } \\
\text { 181 (1838). } \\
\text { Family: Asteraceae }\end{array}$ \\
\hline $\begin{array}{l}\text { 119. L. pinnatifida Cass. } \\
\text { Natural Order: Compositae } \\
\text { Bengal - Not mentioned }\end{array}$ & $\begin{array}{l}\text { 115. Launaea sarmentosa (Willd.) Sch.-Bip. ex } \\
\text { Kuntze, Rev. Gen. Pl. 1: } 350 \text { (1891). Family: } \\
\text { Asteraceae }\end{array}$ \\
\hline $\begin{array}{l}\text { 120. Stylidium kunthii Wall. } \\
\text { Natural Order: Stylidieae } \\
\text { Chittagong - H.f. \& T. }\end{array}$ & $\begin{array}{l}\text { 116. Stylidium kunthii Wall. ex DC., Prodr., 7: } \\
335 \text { (1839). Family: Stylidiaceae }\end{array}$ \\
\hline $\begin{array}{l}\text { 121. S. tenellum Swartz } \\
\text { Natural Order: Stylidieae } \\
\text { Dacca-Clarke and Chittagong - Kurz }\end{array}$ & $\begin{array}{l}\text { 117. Stylidium tenellum Sw., Mag. Ges. Naturf. } \\
\text { Fr. Berlin 1: 51, pl. 2, t. 3, f. } 3 \text { (1807). } \\
\text { Family: Stylidiaceae }\end{array}$ \\
\hline $\begin{array}{l}\text { 122. Lobelia trigona Roxb. } \\
\text { Natural Order: Campanulaceae } \\
\text { Dacca - Clarke }\end{array}$ & $\begin{array}{l}\text { 118. Lobelia alsinoides Lam., Encycl. 3: } 588 \\
\text { (1792). } \\
\text { Family: Campanulaceae }\end{array}$ \\
\hline $\begin{array}{l}\text { 123. L. affinis Wall. } \\
\text { Natural Order: Campanulaceae } \\
\text { Bengal - Not mentioned }\end{array}$ & $\begin{array}{l}\text { 119. Lobelia zeylanica L., Sp. Pl.: } 932 \text { (1753). } \\
\text { Family: Campanulaceae }\end{array}$ \\
\hline $\begin{array}{l}\text { 124. L. terminalis Clarke } \\
\text { Natural Order: Campanulaceae } \\
\text { Mymensingh - Clarke }\end{array}$ & $\begin{array}{l}\text { 120. Lobelia terminalis C.B. Clarke in Hook.f., Fl. } \\
\text { Brit. India 3: } 424 \text { (1881). } \\
\text { Family: Campanulaceae }\end{array}$ \\
\hline $\begin{array}{l}\text { 125. L. rosea Wall. } \\
\text { Natural Order: Campanulaceae } \\
\text { North Bengal - Not mentioned }\end{array}$ & $\begin{array}{l}\text { 121. Lobelia rosea Wall. in Roxb., Fl. Ind. 2: } 115 \\
\text { (1824). } \\
\text { Family: Campanulaceae }\end{array}$ \\
\hline $\begin{array}{l}\text { 126. Campanomoea celebica Blume } \\
\text { Natural Order: Campanulaceae } \\
\text { Chittagong - Not mentioned }\end{array}$ & $\begin{array}{l}\text { 122. Cyclocodon celebicus (Blume) D. Y. Hong, } \\
\text { Acta Phytotax. Sin. 36(2): } 109 \text { (1998). } \\
\text { Family: Campanulaceae }\end{array}$ \\
\hline $\begin{array}{l}\text { 127. Agapetes variegata D. Don } \\
\text { Natural Order: Vacciniaceae } \\
\text { Chittagong hills - Not mentioned }\end{array}$ & $\begin{array}{l}\text { 123. Agapetes variegata (Roxb.) D. Don ex G. } \\
\text { Don, Gen. Hist. 3: } 862 \text { (1834). } \\
\text { Family: Ericaceae }\end{array}$ \\
\hline $\begin{array}{l}\text { 128. A. macrantha Hook.f. } \\
\text { Natural Order: Vacciniaceae } \\
\text { Chittagong hills - Roxburgh }\end{array}$ & $\begin{array}{l}\text { 124. Agapetes macrantha (Hook.) Benth. \& } \\
\text { Hook.f., Gen. Pl. 2: } 571 \text { (1876). } \\
\text { Family: Ericaceae }\end{array}$ \\
\hline $\begin{array}{l}\text { 129. Aegialitis rotundifolia Roxb. } \\
\text { Natural Order: Plumbagineae } \\
\text { Bengal - Not mentioned }\end{array}$ & $\begin{array}{l}\text { 125. Aegialitis rotundifolia Roxb., Fl. Ind. 2: } 111 \\
\text { (1832). } \\
\text { Family: Plumbaginaceae }\end{array}$ \\
\hline
\end{tabular}




\begin{tabular}{|c|c|}
\hline $\begin{array}{l}\text { Species, Natural Order, recorded area - collector's } \\
\text { name/ Wall. Cat. no. as in Hook.f. (1880-1882) }\end{array}$ & $\begin{array}{l}\text { Current nomenclature with loc. cit. and family as of } \\
\text { Cronquist (1981) }\end{array}$ \\
\hline $\begin{array}{l}\text { 130. Lysimachia javanica Bl. } \\
\text { Natural Order: Primulaceae }\end{array}$ & $\begin{array}{l}\text { 126. Lysimachia decurrens G. Forst. in Fl. Ins. } \\
\text { Austr. } 1212 \text { (1786). }\end{array}$ \\
\hline Silhet - Not mentioned & Family: Primulaceae \\
\hline $\begin{array}{l}\text { 131. Anagallis arvensis Linn. } \\
\text { Natural Order: Primulaceae } \\
\text { Bengal - Not mentioned }\end{array}$ & $\begin{array}{l}\text { 127. Anagallis arvensis L., Sp. Pl.: } 148 \text { (1753). } \\
\text { Family: Primulaceae }\end{array}$ \\
\hline $\begin{array}{l}\text { 132. Maesa ramentacea A. DC. } \\
\text { Natural Order: Myrsineae } \\
\text { Eastern Bengal - Not mentioned }\end{array}$ & $\begin{array}{l}\text { 128. Maesa ramentacea (Roxb.) A. DC., Trans. } \\
\text { Linn. Soc. London 17: } 133 \text { (1834). } \\
\text { Family: Myrsinaceae }\end{array}$ \\
\hline $\begin{array}{l}\text { 133. M. paniculata A. DC. } \\
\text { Natural Order: Myrsineae } \\
\text { Silhet (Pundua) - H.f. \& T. }\end{array}$ & $\begin{array}{l}\text { 129. Maesa paniculata A. DC., Trans. Linn. Soc. } \\
\text { London 17: } 133 \text { (1834). } \\
\text { Family: Myrsinaceae }\end{array}$ \\
\hline $\begin{array}{l}\text { 134. Embelia nutans Wall. } \\
\text { Natural Order: Myrsineae } \\
\text { Silhet - Wall. Cat. } 2303 \text { \& H.f. \& T. }\end{array}$ & $\begin{array}{l}\text { 130. Embelia nutans Wall. in Roxb., Fl. Ind. 2: } \\
290 \text { (1824). Family: Myrsinaceae }\end{array}$ \\
\hline $\begin{array}{l}\text { 135. Ardisia paniculata Roxb. } \\
\text { Natural Order: Myrsineae } \\
\text { Dacca -Clarke \& Chittagong - Roxburgh }\end{array}$ & $\begin{array}{l}\text { 131. Ardisia paniculata Roxb., Fl. Ind. 2: } 270 \\
\text { (1824). } \\
\text { Family: Myrsinaceae }\end{array}$ \\
\hline $\begin{array}{l}\text { 136. A. icara Ham. } \\
\text { Natural Order: Myrsineae }\end{array}$ & $\begin{array}{l}\text { 132. Ardisia icara Wall. ex DC., Trans. Linn. Soc. } \\
\text { London 17: } 125 \text { (1834). } \\
\text { Family: Myrsinaceee }\end{array}$ \\
\hline $\begin{array}{l}\text { 137. Amblyanthus glandulosus A. DC. } \\
\text { Natural Order: Myrsineae } \\
\text { Silhet - Wall. Cat. } 2265\end{array}$ & $\begin{array}{l}\text { 133. Amblyanthus glandulosus (Roxb.) A. DC., } \\
\text { Ann. Sci. Nat., Bot. II, 16: 83, t. } 6 \text { (1841). } \\
\text { Family: Myrsinaceae }\end{array}$ \\
\hline $\begin{array}{l}\text { 138. Chrysophyllum Roxburghii G. Don } \\
\text { Natural Order: Sapotaceae } \\
\text { Silhet - Wall. Cat. } 4160\end{array}$ & $\begin{array}{l}\text { 134. Chrysophyllum roxburghii G. Don, Gen. } \\
\text { Hist. 4: } 33 \text { (1837). } \\
\text { Family: Sapotaceae }\end{array}$ \\
\hline $\begin{array}{l}\text { 139. Sideroxylon grandifolium Wall. } \\
\text { Natural Order: Sapotaceae } \\
\text { Silhet - Wall. Cat. 4155, 4156A }\end{array}$ & $\begin{array}{l}\text { 135. Planchonella grandifolia (Wall.) Pierre, Not. } \\
\text { Bot.: } 36 \text { (1890). } \\
\text { Family: Sapotaceae }\end{array}$ \\
\hline $\begin{array}{l}\text { 140. Dichopsis polyantha Benth. } \\
\text { Natural Order: Sapotaceae } \\
\text { Silhet- Wall. Cat. 4166, } 4156 \text { \& } \\
\text { Chittagong - H.f. \& T. }\end{array}$ & $\begin{array}{l}\text { 136. Palaquium polyanthum (Wall. ex G. Don) } \\
\text { Baill in Traite Bot. Med. Phan. } 1500 \text { (1884). } \\
\text { Family: Sapotaceae }\end{array}$ \\
\hline $\begin{array}{l}\text { 141. Diospyros Embryopteris Pers. } \\
\text { Natural Order: Ebenaceae } \\
\text { Bengal - Not mentioned }\end{array}$ & $\begin{array}{l}\text { 137. Diospyros peregrina (Gaertn) Guerke in Nat. } \\
\text { Pflanzenfam. 4(1): } 164 \text { (1891). } \\
\text { Family: Ebenaceae }\end{array}$ \\
\hline $\begin{array}{l}\text { 142. D. Toposia Ham. } \\
\text { Natural Order: Ebenaceae }\end{array}$ & $\begin{array}{l}\text { 138. Diospyros toposia Buch.-Ham.in Trans. Linn. } \\
\text { Soc. London 15: } 115 \text { (1827). }\end{array}$ \\
\hline Silhet \& Chittagong - Roxburgh \& Kurz & Family: Ebenaceae \\
\hline
\end{tabular}




\begin{tabular}{|c|c|}
\hline $\begin{array}{c}\text { Species, Natural Order, recorded area - collector’s } \\
\text { name/ Wall. Cat. no. as in Hook.f. (1880-1882) }\end{array}$ & $\begin{array}{l}\text { Current nomenclature with loc. cit. and family as of } \\
\text { Cronquist (1981) }\end{array}$ \\
\hline 143. D. nigricans Wall. & 139. Diospyros nigricans Wall. ex A. DC., Prodr. \\
\hline Natural Order: Ebenaceae & 8: $239(1844)$ \\
\hline Silhet - Wallich & Family: Ebenaceae \\
\hline 144. D. lanceaefolia Roxb. & 140. Diospyros lanceifolia Roxb., Fl. Ind. 2: 537 \\
\hline Natural Order: Ebenaceae & (1832). \\
\hline Silhet - Roxburgh & Family: Ebenaceae \\
\hline 145. D. stricta Roxb. & 141. Diospyros stricta Roxb., Fl. Ind. 2: 539 \\
\hline Natural Order: Ebenaceae & (1832). \\
\hline Silhet and Comilla - Roxburgh & Family: Ebenaceae \\
\hline 146. D. ramiflora Roxb. & $\begin{array}{l}\text { 142. Diospyros ramiflora Roxb., Fl. Ind. 2: } 535 \\
\text { (1832). }\end{array}$ \\
\hline East Bengal - Roxburgh & Family: Ebenaceae \\
\hline 147. D. elegans Clarke var. Hookeri Clarke & 143. Diospyros elegans C.B. Clarke in Hook.f., Fl. \\
\hline Natural Order: Ebenaceae & Brit. India 3: 571 (1882). \\
\hline Chittagong; Seetakoond - H.f. \& T. & Family: Ebenaceae \\
\hline 148. Symplocos caudata Wall. & 144. Symplocos sumuntia Buch.-Ham. ex D. Don, \\
\hline Natural Order: Styraceae & Prodr. Fl. Nepal. 145 (1825). \\
\hline Chittagong; Seetakoond - H.f. \& T. & Family: Symplocaceae \\
\hline 149. Jasminum sambac Ait. & 145. Jasminum sambac (L.) Sol., Hort. Kew 1: 8 \\
\hline Natural Order: Oleaceae & (1789). \\
\hline Bengal - Not mentioned & Family: Oleaceae \\
\hline 150. J. scandens Vahl & 146. Jasminum scandens (Retz.) Vahl, Symb. Bot. \\
\hline Natural Order: Oleaceae & 3: 2 (1794). \\
\hline Chittagong - Not mentioned & Family: Oleaceae \\
\hline 151. J. anastomosans Wall. & 147. Jasminum nervosum Lour., Fl. Cochinch. 1: \\
\hline Natural Order: Oleaceae & 20 (1790). \\
\hline Silhet; Chattuck - H.f. \& T. & Family: Oleaceae \\
\hline 152. J. anastomosans Wall. var. silhetensis Blume & 147. Jasminum nervosum Lour., Fl. Cochinch. 1: \\
\hline Natural Order: Oleaceae & 20 (1790). \\
\hline Silhet - Not mentioned & Family: Oleaceae \\
\hline 153. J. subtriplinerve Blume & 147. Jasminum nervosum Lour., Fl. Cochinch. 1: \\
\hline Natural Order: Oleaceae & 20 (1790) \\
\hline Silhet - Wallich & Family: Oleaceae \\
\hline 154. J. auriculatum Vahl & 148. Jasminum auriculatum Vahl, Symb. Bot. 3: \\
\hline Natural Order: Oleaceae & 30 (1794). \\
\hline Bengal - Not mentioned & Family: Oleaceae \\
\hline 155. J. lanceolaria Roxb. & 149. Jasminum lanceolaria Roxb., Fl. Ind. 1: 98 \\
\hline Natural Order: Oleaceae & $(1820)$ \\
\hline Jaintea hills - Griffith, H.f. \& T. & Family: Oleaceae \\
\hline
\end{tabular}




\begin{tabular}{|c|c|}
\hline $\begin{array}{l}\text { Species, Natural Order, recorded area - collector’s } \\
\text { name/ Wall. Cat. no. as in Hook.f. (1880-1882) }\end{array}$ & $\begin{array}{c}\text { Current nomenclature with loc. cit. and family as of } \\
\text { Cronquist (1981) }\end{array}$ \\
\hline $\begin{array}{l}\text { 156. Linociera macrophylla Wall. } \\
\text { Natural Order: Oleaceae } \\
\text { Silhet - Wall. Cat. } 2826\end{array}$ & $\begin{array}{l}\text { 150. Chionanthus ramiflorus Roxb., Fl. Ind. 1: } \\
106 \text { (1820). Family: Oleaceae }\end{array}$ \\
\hline $\begin{array}{l}\text { 157. Olea dioica Roxb. } \\
\text { Natural Order: Oleaceae } \\
\text { Chittagong hills - Roxburgh }\end{array}$ & $\begin{array}{l}\text { 151. Olea dioica Roxb., Fl. Ind. 1: } 105 \text { (1820). } \\
\text { Family: Oleaceae }\end{array}$ \\
\hline $\begin{array}{l}\text { 158. Ligustrum robustum Blume } \\
\text { Natural Order: Oleaceae } \\
\text { Silhet, Dacca, Chittagong \& c. } \\
\text { - Not mentioned }\end{array}$ & $\begin{array}{l}\text { 152. Ligustrum robustum (Roxb.) Blume, Mus. } \\
\text { Bot. 1: } 313 \text { (1851). } \\
\text { Family: Oleaceae }\end{array}$ \\
\hline $\begin{array}{l}\text { 159. Myxopyrum smilacifolium Blume } \\
\text { Natural Order: Oleaceae } \\
\text { Silhet and Chittagong - Not mentioned }\end{array}$ & $\begin{array}{l}\text { 153. Myxopyrum smilacifolium (Wall.) Blume, } \\
\text { Mus. Bot. 1: } 320 \text { (1851). } \\
\text { Family: Oleaceae }\end{array}$ \\
\hline $\begin{array}{l}\text { 160. Willoughbeia edulis Roxb. } \\
\text { Natural Order: Apocynaceae } \\
\text { Chittagong - Roxburgh, \& c. }\end{array}$ & $\begin{array}{l}\text { 154. Willoughbeia edulis Roxb., Pl. Corom. 3: } 77 \text {, } \\
\text { t. } 280 \text { (1820). } \\
\text { Family: Apocynaceae }\end{array}$ \\
\hline $\begin{array}{l}\text { 161. Melodinus monogynus Roxb. } \\
\text { Natural Order: Apocynaceae } \\
\text { Silhet - Not mentioned }\end{array}$ & $\begin{array}{l}\text { 155. Melodinus cochinchinensis (Lour.) Merr., } \\
\text { Trans. Amer. Philos. Soc., n.s. 24: } 310 \text { (1935). } \\
\text { Family: Apocynaceae }\end{array}$ \\
\hline $\begin{array}{l}\text { 162. Tabernaemontana recurva Roxb. } \\
\text { Natural Order: Apocynaceae } \\
\text { Chittagong - Roxburgh }\end{array}$ & $\begin{array}{l}\text { 156. Tabernaemontana divaricata (L.) R. Br. ex } \\
\text { Roem. \& Schult., Syst. Veg. 4: } 427 \text { (1819). } \\
\text { Family: Apocynaceae }\end{array}$ \\
\hline $\begin{array}{l}\text { 163. Parsonia spiralis Wall. } \\
\text { Natural Order: Apocynaceae } \\
\text { Silhet - Wall. Cat. 1631, 1632, } 1633\end{array}$ & $\begin{array}{l}\text { 157. Parsonia alboflavescens (Dennst.) Mabb., } \\
\text { Taxon 26: } 532 \text { (1977). } \\
\text { Family: Apocynaceae }\end{array}$ \\
\hline $\begin{array}{l}\text { 164. Vallaris Heynei Spreng. } \\
\text { Natural Order: Apocynaceae } \\
\text { Silhet - Not mentioned }\end{array}$ & $\begin{array}{l}\text { 158. Vallaris solanacea (Roth) Kuntze, Rev. Gen. } \\
\text { Pl. 2: } 417 \text { (1891). } \\
\text { Family: Apocynaceae }\end{array}$ \\
\hline $\begin{array}{l}\text { 165. Pottsia cantonensis Hook. \& Arn. } \\
\text { Natural Order: Apocynaceae } \\
\text { Silhet - De Silva }\end{array}$ & $\begin{array}{l}\text { 159. Pottsia laxiflora (Blume) Kuntze, Rev. Gen. } \\
\text { Pl. 2: } 416 \text { (1891). } \\
\text { Family: Apocynaceae }\end{array}$ \\
\hline $\begin{array}{l}\text { 166. Wrightia coccinea Sims } \\
\text { Natural Order: Apocynaceae } \\
\text { Silhet -Roxburgh, De Silva \& Chittagong - Kurz }\end{array}$ & $\begin{array}{l}\text { 160. Wrightia coccinea (Roxb. ex Hornem.) Sims., } \\
\text { Bot. Mag. 53: t. } 2696 \text { (1826). } \\
\text { Family: Apocynaceae }\end{array}$ \\
\hline $\begin{array}{l}\text { 167. Strophanthus Wallichii A. DC. } \\
\text { Natural Order: Apocynaceae } \\
\text { Chittagong- Seetakoond - H.f. \& T. }\end{array}$ & $\begin{array}{l}\text { 161. Strophanthus wallichii A. DC., Prodr. 8: } 418 \\
\text { (1844). } \\
\text { Family: Apocynaceae }\end{array}$ \\
\hline $\begin{array}{l}\text { 168. Beaumontia grandiflora Wall. } \\
\text { Natural Order: Apocynaceae } \\
\text { Silhet and Chittagong - Not mentioned }\end{array}$ & $\begin{array}{l}\text { 162. Beaumontia grandiflora Wall., Tent. Fl. } \\
\text { Nepal 1: 15, t. } 7 \text { (1824). } \\
\text { Family: Apocynaceae }\end{array}$ \\
\hline
\end{tabular}




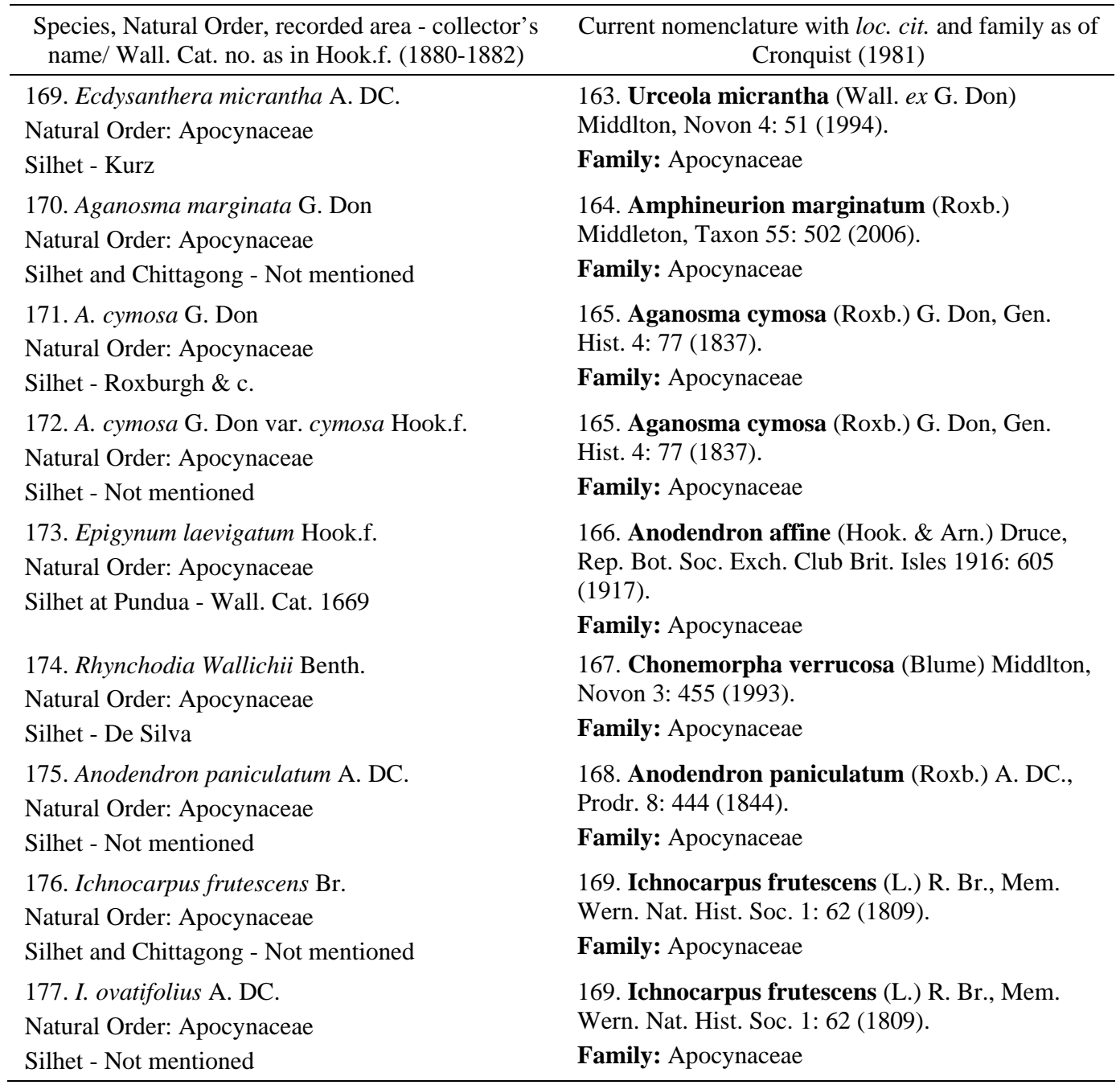

\section{Acknowledgement}

We are thankful to Professor Dr. Md. Abul Hassan, Department of Botany, University of Dhaka for his support to conduct this research.

\section{References}

Ahmed, Z.U., Begum, Z.N.T., Hassan, M.A., Khondker, M., Kabir, S.M.H., Ahmad, M., Ahmed, A.T.A., Rahman, A.K.A. and Haque, E.U. (Eds.). 2008a. Encyclopedia of Flora and Fauna of Bangladesh 6: 1408. Asiatic Society of Bangladesh, Dhaka.

Ahmed, Z.U., Hassan, M.A., Begum, Z.N.T., Khondker, M., Kabir, S.M.H., Ahmad, M., Ahmed, A.T.A., Rahman, A.K.A. and Haque, E.U. (Eds.). 2008b. Encyclopedia of Flora and Fauna of Bangladesh 7: 1546. Asiatic Society of Bangladesh, Dhaka. 
Ahmed, Z.U., Hassan, M.A., Begum, Z.N.T., Khondker, M., Kabir, S.M.H., Ahmad, M., Ahmed, A.T.A., Rahman, A.K.A. and Haque, E.U. (Eds.). 2009a. Encyclopedia of Flora and Fauna of Bangladesh 8: 1478. Angiosperms: Dicotyledons (Fabaceae-Lythraceae). Asiatic Society of Bangladesh, Dhaka.

Ahmed, Z.U., Hassan, M.A., Begum, Z.N.T., Khondker, M., Kabir, S.M.H., Ahmad, M., Ahmed, A.T.A. (Eds.). 2009b. Encyclopedia of Flora and Fauna of Bangladesh 9: 1-488. Asiatic Society of Bangladesh, Dhaka.

Ahmed, Z.U., Hassan, M.A., Begum, Z.N.T., Khondker, M., Kabir, S.M.H., Ahmad, M., Ahmed, A.T.A. (Eds.). 2009c. Encyclopedia of Flora and Fauna of Bangladesh 10: 1-580. Asiatic Society of Bangladesh, Dhaka.

Ali, Z. 1971. Ericaceae. In: Nasir, E. and Ali, S.I. (Eds.), Flora of Pakistan. Fasc. 5: 1- 72. Stewart Herbarium, Gordon College, Rawalpindi, Pakistan.

Brandis, D. 1906. Indian Trees. Bishen Singh Mahendra Pal Singh, Dehra Dun, India- 767pp.

Brumitt, R.K. 1992. Vascular Plants Families and Genera- Royal Botanic Gardens. England, 804 pp.

Brummitt, R.K. and Powell, C.E. 1992. Authors of Plant names. Royal Botanic Gardens, Kew, England, $732 \mathrm{pp}$.

Cowan, J.M. 1926. The Flora of Chakaria Sundarbans. Rec. Bot. Surv. Ind. 11(2): 197-225.

Cronquist, A. 1981. An Integrated System of Classification of Flowering Plants. Columbia University Press$1262 \mathrm{pp}$.

Hara, H. and Williams, L.H.J. 1979. An Enumeration of the Flowering Palnts of Nepal 2: 1 - 220. Trustee of British Museum (Natural history), London.

Hara, H., Chater, O.A. and Williams, L.H.J. 1982. An Enumeration of the Flowering Plants of Nepal. 3: 1-226. Trustee of British Museum (Natural history), London.

Heinig, R.L. 1925. List of Plants of Chittagong Collectorate and the Hill Tracts - The Bengal Government Branch Press, Darjeeling, India, 84 pp.

Hooker, J.D. 1880-1882. The Flora of British India 3: 1-712. L. Reeve \& Co. Ltd., Kent, England.

Internet sources: I. The Plant List, KEW II. IPNI for plant name query III. Google search: plant names and others

Ishaq, M. 1979. Bangladesh Gazetteers, Ministry of Cabinet Affairs, Establishment Division, Bangladesh Government press, Dhaka, Bangladesh.

Kanjilal, U.N., Das, A., Kanjilal, P.C. and De, R.N. (Eds.) 1939. Flora of Assam. 3: 1-578. Government of Assam, Shillong, India.

Kurz, W.S. 1877. Forest Flora of British Burma, 2: 1-550. Reprt.,1974. Bishen Sing Mahendra Pal Singh, Dhera Dun - India.

Mabberley, D.J. 1997. The Plant-Book, a portable dictionary of the vascular plants- (2nd edition). Cambridge University Press, Cambridge, UK- 858 pp.

McNeill, J., Barrie, F.R., Buck, W.R., Demoulin, V., Greuter, W., Hawksworth, D.L., Herendeen, P.S., Knapp, S., Marhold, K., Prado, J., Prudhomme Van Reine, W.F., Smith, G.F., Wiersema, J.H. and Turland, N.J. (Eds.). 2012. International Code of Nomenclature for algae, fungi, and plants (Melbourne Code), Adopted by the Eighteenth International Botanical Congress Melbourne, Australia, July 2011 (electronic ed.). Bratislava: International Association for Plant Taxonomy. Regnum Vegetabile, 154. Koeltz Scientific Books, Melbourne, Australia.

Prain, D. 1903. Bengal Plants, I: 1-490; II: 491-1013. Botanical Survey of India.

Press, J.R., Shrestha, K.K. and Sutton, D.A. 2000. Annotated Checklist of the Flowering Plants of Nepal. The Natural History Museum, London- 430 pp.

Rahman, M.A. and Wilcock, C.C. 1991. Periplocaceae In: Khan, M.S. and Rahman, M.M. (Eds.) Flora of Bangladesh. Fasc. 47: 1-16. Bangladesh National Herbarium, Dhaka.

Rahman, M.A. and Wilcock, C.C. 1995. Asclepiadaceae In: Khan, M.S. and Rahman, M.M. (Eds.) Flora of Bangladesh. Fasc. 48: 1-71. Bangladesh National Herbarium, Ministry of Environment and Forest, Dhaka. 
Raizada, M.B. 1941. On the flora of Chittagong. The Indian Forester 67(5): 245-254.

Rashid, M.E. and Rahman, M.A. 2011. Updated nomenclature and taxonomic status of the plants of Bangladesh included in Hook.f., the Flora of British India: Volume-I. Bangladesh J. Plant Taxon. 18(2): 177-197.

Rashid, M.E. and Rahman, M.A. 2012. Updated nomenclature and taxonomic status of the plants of Bangladesh included in Hook.f., the Flora of British India: Volume-II. Bangladesh J. Plant Taxon. 19 (2): 173-190.

Roxburgh, W. 1814. Hortus Bengalensis. Boerhaave Press, Leiden (Holland). pp.1-105.

Roxburgh, W. 1820. Flora Indica. Carey, W. and Wallich, N. (Eds.) Vol. 1. Mission Press, Serampore, Calcutta, India.

Roxburgh, W. 1824. Flora Indica. Carey, W. and Wallich, N. (Eds.) Vol. 2. Mission Press, Serampore, Calcutta, India.

Roxburgh, W. 1832. Flora Indica. Carey, W. (Ed.) Vol. 3. Mission Press, Serampore, Calcutta, India.

Sinclair, J. 1956. The Flora of Cox’s Bazar, East Pakistan. Bull. Bot. Soc.Beng. 9(2): 1-116.

Voss, E.G. (Ed.) 1983. International Code of Botanical Nomenclature (ICBN), Regnum Vegetabile, Vol. 97. Bohn, Scheltema \& Holkema, Utecht.

Wallich, N. 1828-1849. A Numerical list of dried specimens of plants in the East Indian Company's Museum, Ined.

Wu, Z.Y. and Raven, P.H. (Eds.). 1994. Flora of China- Vol. 17 (Verbenaceae through Solanaceae). Science Press, Beijing, and Missouri Botanical Garden Press, St. Louis.

Wu, Z.Y., Raven, P. H and Hong, D.Y. (Eds.). 2005. Flora of China- Vol. 14 (Apiaceae through Ericaceae). Science Press, Beijing, and Missouri Botanical Garden Press, St. Louis.

(Manuscript received on 10 March 2016; revised on 10 November 2016) 